\title{
Analysis of the microstructure of the fuel spray atomized by marine injector
}

The process of the atomization and formation of the fuel spray can be described by macro- and microstructure parameters. Knowledge of these parameters may be a key information to conduct further optimization of the combustion process. This paper presents the research results of the microstructure parameters of the diesel oil spray atomized with marine engine injector. A measurement technique, named Laser Induced Fluorescence (LIF) in the combination with Mie scattering was used to determine LIF/Mie ratio across the spray. The fuel was injected into a constant volume vessel. LIF and Mie signals were recorded by two cameras at the same time. Nd:YAG pulsed laser was used to create light sheet for spray illumination. Wavelength of $\lambda=266 \mathrm{~nm}$ was used in this study.

Key words: marine diesel engine, marine injector, microstructure spray parameters, optical method, LIF/Mie

\section{Introduction}

Diesel engines are the main type of drive for large-sized marine vessels. Burning diesel oil by marine engines effects the emission of toxic compounds into the atmosphere. The emissions generated by engine, similar to a marine vessel engine, depending on various design feature are reported in the literature [1-3]. The amount of exhaust emissions are regulated and gradually tightened by International Maritime Organization (IMO) MARPOL 73/78 regulations, attachment VI including chapter 4 [4]. Stricter exhaust emission regulations are the main reason for optimizing the combustion process. The process of designing the marine engine and its units is aimed at maximizing engine efficiency while maintaining low emission of toxic compounds. High pressure of injection, controlled fuel delivery, and increased engine effectiveness are the advantages of the common rail system. Nevertheless, there is enormous number of engines equipped with conventional pressure-opened injectors. Retrofitting these engines with CR injection system in order to meet stricter emissions standards in most cases is not economically feasible. Therefore, more cost-effective method to optimize combustion process in engines in operation needs to be implemented.

The combustion of fuel in the combustion chamber of marine engine begins at certain time after start of injection. This time is necessary for creating the fuel-air mixture of proper parameters. This process is dependent on atomization, evaporation and diffusion. Payri et al. [5] defined five phases of creating combusting fuel spray: I) non-reacting, II) auto-ignition expansion, III) stabilization, IV) acceleration, and V) quasi-steady propagation. First phase contains atomization which includes primary and secondary brakeup [6]. The next following ones are strongly dependent on the first one, therefore injection process is of crucial importance. Atomization occurs as a result of internal turbulences (induced at inlet to the nozzle and by cavitation) and aerodynamic forces.

Creating proper macrostructure and microstructure [7] of diesel fuel spray may lead to decreased exhaust emissions and increased efficiency.

Injected fuel spray takes the shape of a cone with a spherical forehead described with parameters: tip penetration and spray cone angle. Tip penetration and spray cone angle demonstrate droplets ability to penetrate gaseous environment in the combustion chamber. Droplets in the fuel spray are usually non-homogeneous in terms of size, which is characterized by a large spread of droplet diameters. Droplets diameters in the fuel spray define the microstructure [6,8-10]. The size of fuel droplets depends on many factors, so the process of their formation is often referred to as the statistical process - the spray spectrum [7]. The most representative parameter used to assess spray quality is the mean diameter of the droplets. The average diameter of the droplets refers to number and diameter, surface or volume of droplets in the fuel spray. The most commonly used is the SMD - Sauter Mean Diameter [11]. SMD may be directly connected to evaporation and combustion of fuel droplets.

Recent research shows that the structure of the diesel fuel spray is influenced by the construction and technical condition of the injection system or the pressure in the combustion chamber $[9,12]$. Studies presented in $[13,14]$ contain the comparison of fuel spray parameters obtained with piezoelectric and solenoid injector. The influence of injection pressure on fuel spray parameters is shown in [15], while backpressure in [16, 17]. The nozzle parameters influence are shown in [18]. While considering the fuel spray parameters, it is necessary to mention cavitation phenomena [19-21]. The increase in injection pressure and the cavitation effect cause a decrease of SMD [22]. Thus, the cavitation may improve quality of the combustion process.

One may notice that the spray formation process can be improved by changing number of parameters, which does not necessarily need to require high investments. The results of the modification need to be properly measured. Diesel spray however, pose a challenge in terms of droplet diameter measurements. Due to small droplets and high optical density of the diesel spray the number of techniques which can be used for spray microstructure characterization is limited. One of the methods is ballistic imaging [23-25]. This technique in a form of optical shadowgraphy was used to visualize breakup of the near-field of an atomizing Diesel spay by Linne et al. [23]. The other possible methods are based on X-ray absorption [26]. The technique which can provide SMD distribution across the spray cloud is LIF/Mie method - also called Laser Sheet Dropsizing (LSD). Pastor et al. [27] used this method to characterize droplet size 
distribution in Diesel spays at three different engine relevant discharge densities. They conducted experiments using two different light sheet illumination wavelengths $355 \mathrm{~nm}$ and $532 \mathrm{~nm} .355 \mathrm{~nm}$ signal was used to induce natural fluorescence of Diesel fuel while $532 \mathrm{~nm}$ signal was used to induce fluorescence of rhodamine B doped to Diesel fuel. They noticed dissymmetrical behavior of natural fluorescence signal. The signal emitted by the Rhodamine B didn't reveal such behavior. They concluded that the observed dissymmetrical behavior of the natural fluorescence signal made this solution unreliable for quantifying the droplet diameter via the LIF/Mie ratio, and indicated second approach as appropriate one. In this study, we used LIF/Mie method to quantify SMD distribution across the marine injector spray at engine relevant pressures. Fluorescence signal in our study was induced at $266 \mathrm{~nm}$ in order to overcome dissymmetrical behavior reported by Pastor et al. [27].

\section{Experimental setup}

Laboratory studies presented in this article were carried out at Warsaw University of Technology. The constant volume vessel was used to observe sprays at elevated pressure. constant volume vessel is shown in Fig. 1.

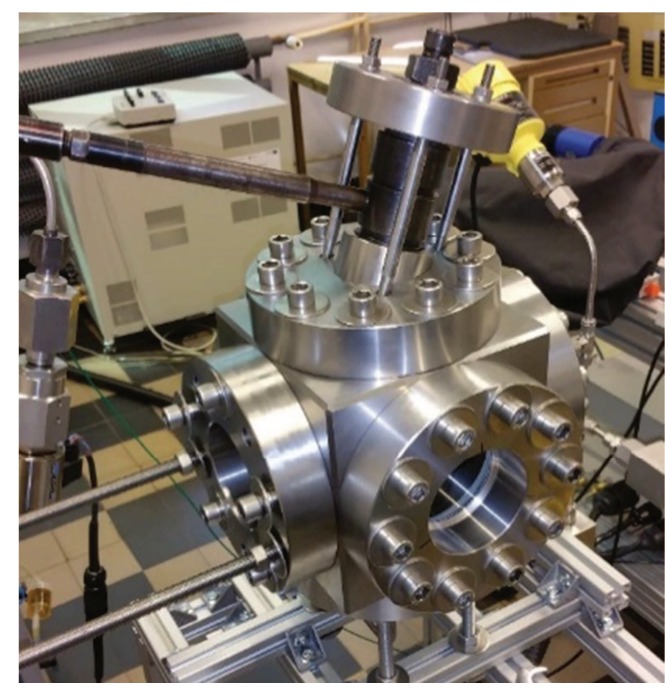

Fig. 1. Constant volume vessel with injector on the top

The parameters of the combustion vessel were summarized in Table 1.

Table 1. The parameters of constant volume vessel

\begin{tabular}{|l|l|l|}
\hline Dimensions & $200 \times 200$ & $\mathrm{~mm}$ \\
\hline Optical view & 100 & $\mathrm{~mm}$ \\
\hline Under pressure & 3.2 & $\mathrm{MPa}$ \\
\hline Measurement space & nitrogen & - \\
\hline Number observation windows & 3 (all 4) & - \\
\hline
\end{tabular}

The injector used in this study is a conventional pressure opened diesel injector from marine engine Sulzer Al 25/30 type, equipped with UPS (Unit pump system) injection system. The injector was located at the top of the vessel in a way to observe single jet evolution at a distance of almost $100 \mathrm{~mm}$ (half of the cylinder bore). The tested spray of diesel oil was made by one active injector nozzle. Rest of the nozzles was plugged. The diameter of the nozzle was
$0.320 \mathrm{~mm}$. The fuel was supplied to the injector by a high pressure common rail system equipped with fast-acting electromagnetic valve releasing fuel flow to the injector. The pressure behind the injector was measured by means of Kistler type 4067E [28] piezorezistive pressure sensor. The specifications of the pressure sensor are shown in Tab. 2.

Table 2. Kistler type 4067E pressure transducer specifications according to [28]

\begin{tabular}{|l|l|}
\hline Parameter & Value \\
\hline Measuring range $/ \mathrm{MPa}$ & $0-300$ \\
\hline Overload $/ \mathrm{MPa}$ & $350 \mathrm{MPa}$ \\
\hline Reference temperature $\left(\mathrm{T}\right.$ ref) $/{ }^{\circ} \mathrm{C}$ & 25 \\
\hline Sensor temperature, min. $/ \mathrm{max} . /{ }^{\circ} \mathrm{C}$ & $-40 / 200$ \\
\hline Temperat. compensation range $/{ }^{\circ} \mathrm{C}$ & $25-180$ \\
\hline Max. deviation pressure $/ \% \mathrm{FSO}$ & $\leq \pm 0.8$ \\
\hline Max. deviation temperature $/{ }^{\circ} \mathrm{C}$ & $\leq \pm 4$ \\
\hline Linearity at Tref $(\mathrm{LSQ}) / \% \mathrm{FSO}$ & $\leq \pm 0.3$ \\
\hline Natural frequency $/ \mathrm{kHz}$ & $>200$ \\
\hline
\end{tabular}

The fuel pressure in common rail system was of 50 $\mathrm{MPa}$. The opening pressure was set to $35 \mathrm{MPa}$. The pressure in the vessel was of $3.2 \mathrm{MPa}$. Instead of air, pure nitrogen was used. The research was conducted at room temperature. Each measurement point was repeated 10 times.

Measured diesel oil viscosity and density at $40^{\circ} \mathrm{C}$ was of $2.35 \mathrm{mPa} \cdot \mathrm{s}$ and $816.1 \mathrm{~kg} / \mathrm{m}^{3}$ respectively.

In this work, the laser-based system was used for the purpose of simultaneous imaging of fluorescence and Mie scattering. The laser beam was generated by SpectraPhysics Nd:YAG pulsed laser of the maximal energy of the signal $220 \mathrm{~mJ}$ (at $266 \mathrm{~nm}$ ) [29]. Generated fuel spray was illuminated by a collimated laser beam. The lights sheet height was of $50 \mathrm{~mm}$.

The experimental setup system is shown in Fig. 2.

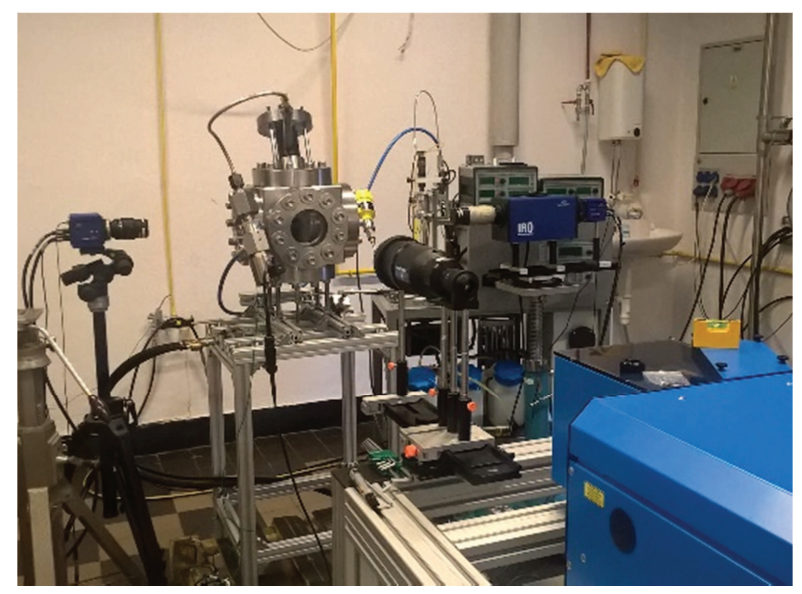

Fig. 2. Experimental setup

The intensity of the signal of LIF and Mie was registered simultaneously with two video cameras SCMOS manufactured by La Vision. The parameters of the video cameras were presented in Tab. 3 . 
Table 3. The parameters of sCMOS Camera

\begin{tabular}{|l|l|l|}
\hline Frequency & 10 & $\mathrm{~Hz}$ \\
\hline Min. exposure & 15 & $\mu \mathrm{s}$ \\
\hline Spectral range & $370-1100$ & $\mathrm{~nm}$ \\
\hline Number of pixels & $2560 \times 2160$ & Pixels \\
\hline Pixel size & $6.5 \times 6.5$ & $\mu \mathrm{m}$ \\
\hline Active area & $16.6 \times 14.0$ & $\mathrm{~mm}$ \\
\hline A/D converter & 16 & bit \\
\hline
\end{tabular}

The cameras were set oppositely in one axis (Fig. 3), directed towards illuminated plane of diesel spray. The signals of LIF and Mie were separated with the adequate optical filters. In order to capture $266 \mathrm{~nm}$ Mie signal the videocamera registering the image of Mie signals was coupled with the IRO La Vision intensifier. IRO caused the intensification of Mie signal before it was projected onto the matrix of camera sCMOS La Vision.

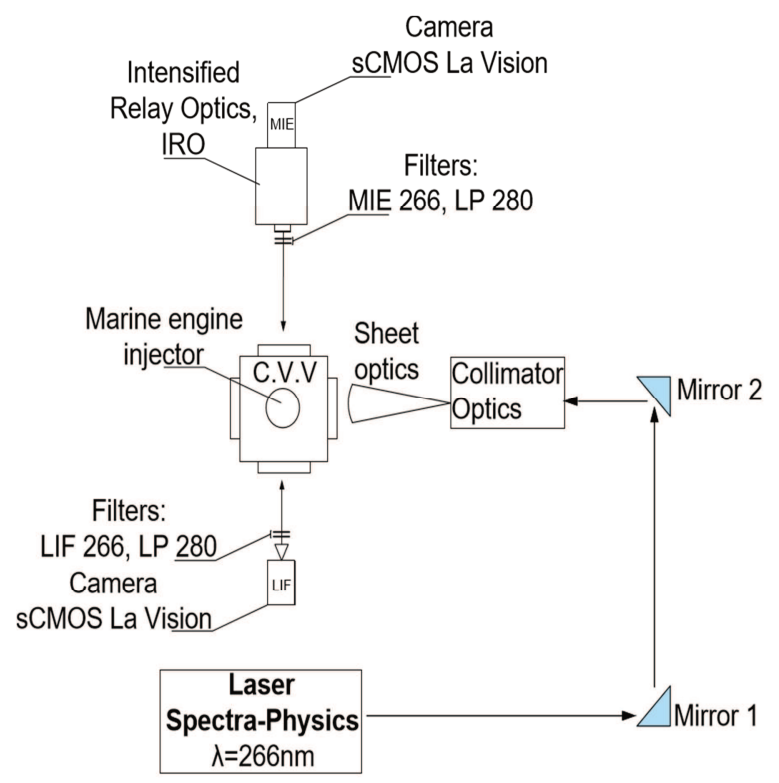

Fig. 3. Experimental setup of the LIF/Mie optical arrangement, C.V.V Constant Volume Vessel

\section{Results}

The principle of LIF/Mie technique is based on the assumption that the intensity of scattered light on the surface of the droplets is proportional to their surface, while the fluorescence intensity is proportional to their volume. The ratio of the intensity of fluorescence to Mie signal therefore represents the Sauter Mean Diameter (SMD). Note, that this is true for spherical droplets, thus the spray region close to the nozzle, where ligaments and strongly deformed droplets are present, cannot be characterized with this technique. One needs to be aware that LIF/Mie ratio are not correlated linearly with SMD as explained by Berrocal et al. [30]. Another important aspect of using laser sheet imaging techniques for dense sprays is multiple scattering. According to Berrocal et al [30] in non-reacting diesel spray usually more than $65 \%$ of the received signal may come from multiple scattering. Therefore, the results presented here shall be treated as qualitative ones.

As a result of the measurements, we received LIF and Mie diesel spray images. Using the appropriate image pro- cessing allows obtaining LIF/Mie ratio 2D map that serves as the basis to determine SMD.

Before acquisition of LIF and Mie signals coming from the illuminated spray at first background and laser light sheet images were taken. These were important in proper image processing. The background and light sheet images are shown in Fig. 4. Note that all images presented here are shown using the same colormap, however the range of the scale differs depending on the image.

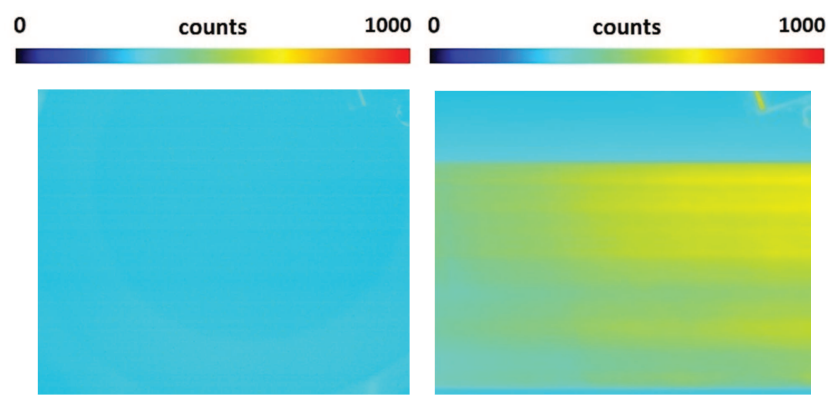

Fig. 4. Background and light sheet images, respectively from left

The first step of results processing included background subtraction from acquired raw LIF and Mie images. Instantaneous LIF and Mie images recorder during one of the injections after background subtraction are shown in Fig. 5 and Fig. 6 respectively.

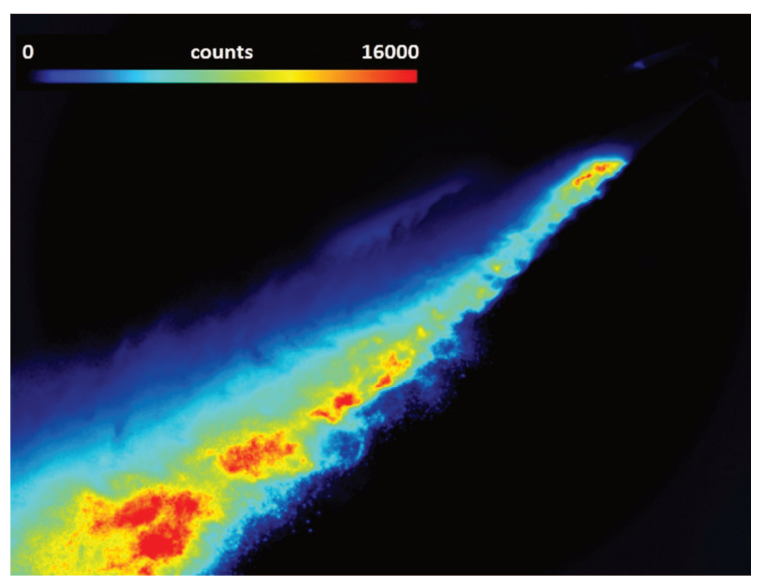

Fig. 5. Instantaneous LIF image after background subtraction

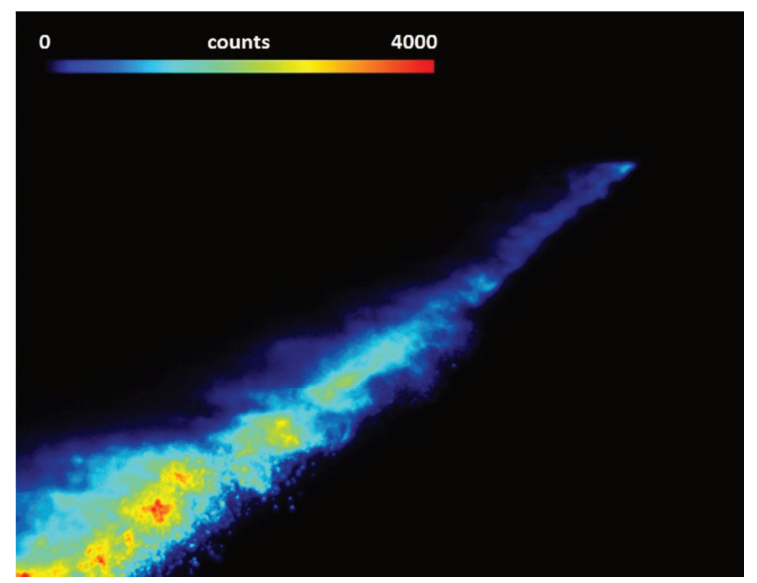

Fig. 6. Instantaneous Mie image after background subtraction 
10 instantaneous images for each signal after background subtraction were averaged. The averaged images of LIF and Mie signal are shown in Fig. 6 and Fig. 7 respectively.

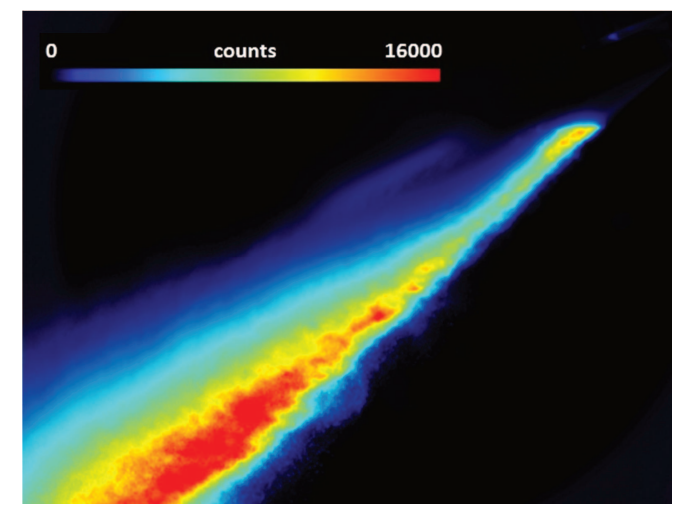

Fig. 7. Instantaneous LIF image after background subtraction

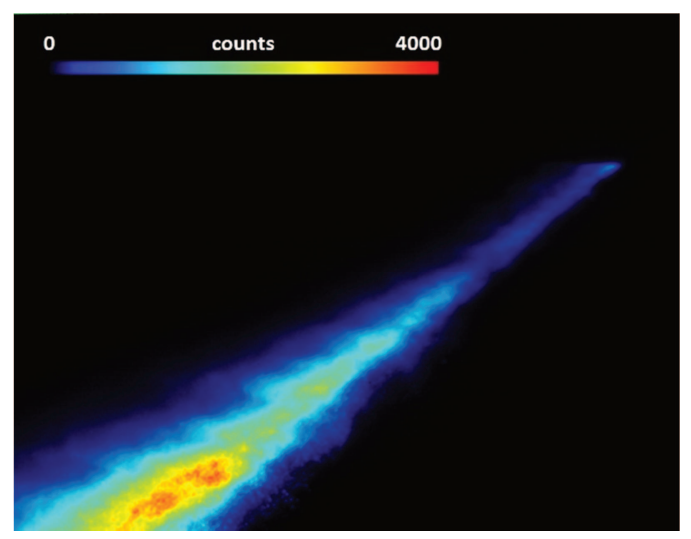

Fig. 8. Instantaneous Mie image after background subtraction

As explained earlier only 10 instantaneous images were used to create average image. The averaged images are still influenced by unsteady turbulent structures what indicates that the number of images should be higher in order to create smooth LIF and Mie images. Nevertheless, comparing to instantaneous images the unsteady turbulent structures were strongly limited. The averaged images were used to create LIF/Mie ratio 2D map which is shown in Fig. 9. LIF/Mie ratio shown in Fig. 9. was multiplied by 100.

Superimposing the images of Mie and LIF signals results in the visualization of created liquid and gas phase in the diesel spray. Basing on received visualizations, it is possible to determine the shape of diesel spray. The analysis of drops distribution in the gas and liquid phase is a quite important aspect in case of the marine internal combustion engine because it is connected to the quality of created air-fuel mixture and the ignition process. Fig. 7. and Fig. 8. show that the intensity of signal decreases in a radial direction outside the diesel spray cone. Reduction of signal intensity proclaims the decrease in density of drops distribution and a decrease in diameter. It is important to remember that the diesel spray was created under back pressure conditions - 3.2 MPa. The back pressure in the constant volume vessel contributes to increase in appearing aerodynamic forces and at the same time to the breakup of the drops. The highest concentration occurs in the axial part, which indicates the presence of a liquid phase and a dense distribution of droplets. The images of LIF signal (Fig. 7) show high intensity in the middle part of a spray cone and at a minor distance from the nozzle. However, Mie images (Fig. 8) present significant intensity at the end of the spray cone and relatively small intensity at the short distance from the nozzle. LIF imaging through the fluorescence excited by the laser light wave may lead to obtaining the fluorescent signal in the gas part. The involvement of fluorescence from the gas phase should be minimal.

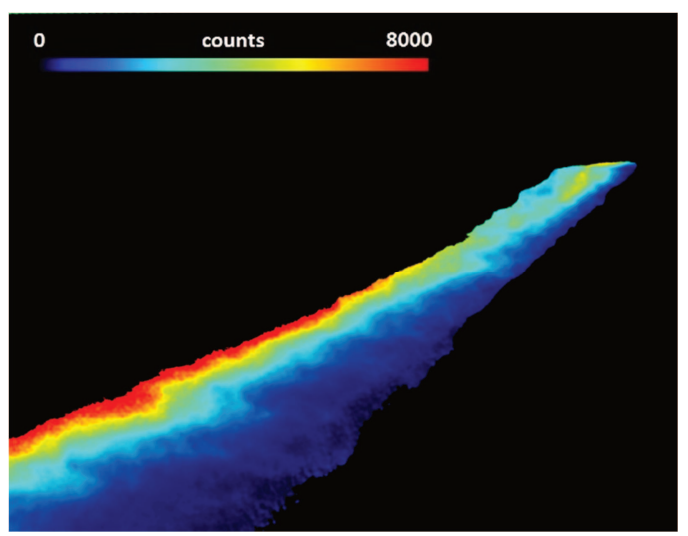

Fig. 9. LIF/Mie ratio (based on averaged images)

\section{Conclusions}

The results presented here show that the dissymmetrical behaviour of natural frequency concerns also $266 \mathrm{~nm}$ excitation wavelength. Mie signal in distinguish to LIF signal was much more symmetrical. Nevertheless, the ratio of LIF/Mie was strongly dissymmetrical and could not be used for SMD determination. The determined $\mathrm{LIF} / \mathrm{Mie}$ ratio suggests that large droplets are on one side of the spray while the small ones are on the opposite side, which is untrue.

Although the results obtained here cannot be used to conclude on droplet size distribution they are highly valuable. It is proven here that dissymmetrical behavior of the natural fluorescence, noticed by Pastor et al [27] for $355 \mathrm{~nm}$ wavelength, cannot be overcome by switching from $3^{\text {rd }}$ to $4^{\text {th }}$ harmonic of Nd:YAG laser. This in turn indicate that the proper approach to SMD determination in Diesel sprays shall be done using rhodamine B, as proposed by Pastor et al. [27].

\section{Acknowledgements}

The authors would like to thank Ph.D., D.Sc., Eng., Jerzy Kowalski Gdynia Maritime University and M.Sc., Eng., Piotr Jaworski Warsaw University of Technology for their technical support and valuable remarks to the optical research of the fuel injection parameters.

\section{Nomenclature}




\section{Bibliography}

[1] SARVI, A., FOGELHOLM, C.J., ZEVENHOVEN, R. Emissions from large-scale medium-speed diesel engines: 1. Influence of engine operation mode and turbocharger. Fuel Process Technol. 2008, 89, 510-519.

[2] SARVI, A., FOGELHOLM, C.J., ZEVENHOVEN, R. Emissions from large-scale medium-speed diesel engines: 2. Influence of fuel type and operating mode. Fuel Process Technol. 2008, 89, 520-527.

[3] SARVI, A., KILPINEN, P., ZEVENHOVEN, R. Emissions from large-scale medium-speed diesel engines: 3 . Influence of direct water injection and common rail. Fuel Process Technol. 2009, 90, 222-231.

[4] IMO. IMO Marine Engine Resolutions, Greenhouse Gas Emissions 2017.

[5] PAYRI, R., GARCÍA-OLIVER, J.M., XUAN, T., BARDI, M. A study on diesel spray tip penetration and radial expansion under reacting conditions. Appl Therm Eng. 2015, 90, 619-629.

[6] NAGASAKA, K., TAKAGI, T., KOYANAGI, K., YAMAUCHI, T. Development of fine atomization injector. JSAE Rev. 2000, 21, 309-313.

[7] Orzechowski Z. Wytwarzanie i zastosowanie rozpylonej cieczy. Wydawnictwo Naukowo Techniczne. 2008.

[8] JU, D., JIA, X., HUANG, Z. et al. Comparison of atomization characteristics of model exhaust gas dissolved diesel and gasoline. Fuel. 2016, 182, 928-934.

[9] WANG, Z., JIANG, C., XU, H., WYSZYNSKI, M.L. Macroscopic and microscopic characterization of diesel spray under room temperature and low temperature with split injection. Fuel Process Technol. 2016, 142, 71-85.

[10] PARK, S., WOO, S., KIM, H., LEE, K. The characteristic of spray using diesel water emulsified fuel in a diesel engine. Appl Energy. 2016, 176, 209-220.

[11] MISHRA, Y.N., KRISTENSSON, E., BERROCAL, E. Reliable LIF/Mie droplet sizing in sprays using structured laser illumination planar imaging. Opt Express. 2014, 22, 4480.

[12] LI, D., GAO, Y., LIU, S. et al. Effect of polyoxymethylene dimethyl ethers addition on spray and atomization characteristics using a common rail diesel injection system. Fuel. 2016, 186, 235-247.

[13] YU, W., YANG, W., TAY, K. et al. Macroscopic spray characteristics of kerosene and diesel based on two different piezoelectric and solenoid injectors. Exp Therm Fluid Sci. 2016, 76, 12-23.

[14] SUH, H.K., PARK, S.W., LEE, C.S. Effect of piezo-driven injection system on the macroscopic and microscopic atomization characteristics of diesel fuel spray. Fuel. 2007, 86, 2833-2845.

[15] LEE, S., PARK, S. Spray atomization characteristics of a GDI injector equipped with a group-hole nozzle. Fuel. 2014 , 137, 50-59.

[16] PAYRI, R., VIERA, J.P., GOPALAKRISHNAN, V., SZYMKOWICZ, P.G. The effect of nozzle geometry over
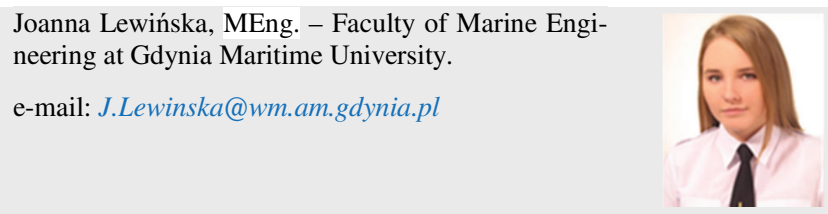

the evaporative spray formation for three different fuels. Fuel. 2017, 188, 645-660.

[17] PAYRI, R., SALVADOR, F.J., GIMENO, J., DE LA MORENA, J. Effects of nozzle geometry on direct injection diesel engine combustion process. Appl Therm Eng. 2009, 29, 2051-2060.

[18] PAYRI, R., SALVADOR, F.J., GIMENO, J., ZAPATA, L.D. Diesel nozzle geometry influence on spray liquid-phase fuel penetration in evaporative conditions. Fuel. 2008, 87, 1165-1176.

[19] BADOCK, C., WIRTH, R., FATH, A., LEIPERTZ, A. Investigation of cavitation in real size diesel injection nozzles. Int J Heat Fluid Flow. 1999, 20, 538-544.

[20] SOU, A., HOSOKAWA, S., TOMIYAMA, A. Effects of cavitation in a nozzle on liquid jet atomization. Int $J$ Heat Mass Transf. 2007, 50, 3575-3582.

[21] DESANTES, J.M., PAYRI, R., SALVADOR, F.J., DE LA MORENA, J. Influence of cavitation phenomenon on primary break-up and spray behavior at stationary conditions. Fuel. 2010, 89, 3033-3041.

[22] SUH, H.K., LEE, C.S. Effect of cavitation in nozzle orifice on the diesel fuel atomization characteristics. Int J Heat Fluid Flow. 2008, 29, 1001-1009.

[23] LINNE, M., PACIARONI, M., HALL, T., PARKER, T. Ballistic imaging of the near field in a diesel spray. Exp Fluids. 2006, 40, 836-846.

[24] LINNE, M.A., PACIARONI, M., BERROCAL, E., SEDARSKY, D. Ballistic imaging of liquid breakup processes in dense sprays. Proc Combust Inst. 2009, 32 II, 2147-2161.

[25] DURAN, S.P., PORTER, J.M., PARKER, T.E. Ballistic imaging of diesel sprays using a picosecond laser: characterization and demonstration. Appl Opt. 2015, 54, 1743.

[26] POWELL, C.F., CIATTI, S.A., CHEONG, S.-K. et al. X-ray absorption measurements of diesel sprays and the effects of nozzle geometry. SAE Technical Paper. SAE International; 2004.

[27] PASTOR, J.V., PAYRI, R., SALAVERT, J.M., MANIN, J. Evaluation of natural and tracer fluorescent emission methods for droplet size measurements in a diesel spray. Int J Automot Technol. 2012, 13, 713-724.

[28] Kistler. Piezoresistive high pressure sensor 2014. www.kistler.com/?type $=669 \&$ fid $=61054 \&$ model $=$ document\&callee $=$ frontend $($ accessed March 5, 2017).

[29] KAPUSTA, Ł.J., JAWORSKI, P., TEODORCZYK, A., KOWALSKI, J. Laser based diagnostic system for spray measurements. J KONES Powertrain Transp. 2015, 22, 91-98.

[30] BERROCAL, E., KRISTENSSON, E., HOTTENBACH, P. et al. Quantitative imaging of a non-combusting diesel spray using structured laser illumination planar imaging. Appl Phys B. 2012, 109, 683-694.

Łukasz Jan Kapusta, DEng. - Faculty of Power and Aeronautical Engineering at Warsaw University of Technology.

e-mail: Lukasz.Kapusta@itc.pw.edu.pl 